\title{
Zur intraspezifischen Lautkommunikation des Russischen Desmans Desmana moschata (Linnaeus, 1758) (Insectivora: Talpidae: Desmaninae) nebst einigen Angaben zu seinem Sozialverhalten
}

\author{
Peter Romanow ${ }^{1}$, Walter Poduschka ${ }^{2}$ \& Werner Deutsch ${ }^{3}$ \\ ${ }^{1}$ Damals Mitarbeiter der Wissenschaftlichen Akademie der ehemaligen UdSSR, Institut für Tiermorphologie \\ und Ökologie, Leninski Prospect, Moskau; ${ }^{2}$ Universität Wien, Institut für Zoologie, 1090 Wien, Althanstraße \\ 9 und Institut für Psychologie, Liebiggasse 5, 1010 Wien; ${ }^{3}$ Österreichische Akademie der Wissenschaften, \\ Institut für Schallforschung, Liebiggasse 5, 1010 Wien
}

Keywords: Russian Desman, Desmana moschata, acoustic communication, sonagrams, Oka Reserve, female dominance, restricted social tolerance

\begin{abstract}
The Russian Desman Desmana moschata (Linnaeus, 1758) is a highly endangered semiaquatic mammal, confined to disjunct areas. Between 1983 and 1992 several specimens were kept and investigated, firstly in the zoological garden of Moscow (4 q \%, $10^{\circ}$ ), and afterwards in the field station of the former SovietRussian Academy of Sciences at Cernogolovka (1\%, $\left.40^{\circ} 0^{\circ}\right)$. Parallel to this, numerous extended field trips to the huge wetlands of the Oka Reserve allowed the verification of the results obtained in captivity.

An unexpectedly rich repertoire of acoustic signals was recorded and transferred to sonagrams in Vienna/Austria. After tentative interpretation, the acoustic signals were correlated with simultaneous behaviour patterns. Not only the essential importance of intraspecific vocalizations was shown, but also a fascinating versatility and variability of the phonetical signals was discovered.

Contrary to the notorious unsociableness of most insectivores, Desmana moschata is different. In this respect it is probably similar to another semiaquatic talpid, Condylura cristata Linnaeus, 1758. Throughout the year, both sexes of Desmana moschata may stay together, provided there is only one adult female present. In this set-up, even an added male newcomer is tolerated, provided he keeps out of the way for a couple of days. Generally, females seem to be absolutely dominant, which appears from their regular territorial dominance.
\end{abstract}

\section{Zusammenfassung}

Der Russische Desman Desmana moschata (Linnaeus, 1758) ist ein nur mehr in disjunkten Gebieten lebendes und deshalb umso gefährdeteres, semiaquatiles Säugetier. Daher wurden zwischen 1983 und 1992 zuerst mehrere Individuen im Moskauer Zoo $\left(4 \%\right.$, $\left.10^{\circ}\right)$ und später in der Feldstation der Akademie der
Wissenschaften der ehemaligen Sowjet-Union in Cernogolovka $\left(1 \%, 40^{\circ} \sigma^{\circ}\right)$ gehalten und beobachtet. Parallel dazu wurden zahlreiche ausgedehnte Freilanduntersuchungen in den weitläufigen Feuchtgebieten der Oka Reserve durchgeführt, um die in den genannten Haltungssystemen gewonnenen Ergebnisse zu überprüfen.

Das erstaunlich reichhaltige Lautrepertoire wurde auf Tonband aufgenommen und in Wien/Österreich auf Sonagramme überspielt. Nach versuchsweiser Deutung der akustischen Signale wurden diese mit simultanen Verhaltensweisen korreliert. Nicht nur die generelle Wichtigkeit der intraspezifischen Lautgebung, sondern auch ein faszinierendes Spektrum akustischer Kommunikationsmöglichkeiten konnten festgestellt werden.

Im Gegensatz zur bekannten Ungeselligkeit der meisten Insectivora hat Desmana moschata ein anderes Sozialsystem, wie es von einem weiteren semiaquatilen Talpiden, Condylura cristata Linnaeus, 1758, bekannt ist. Während des ganzen Jahres können beide Geschlechter von Desmana moschata in einem Sozialverband leben, soferne unter ihnen nur ein einziges adultes $\odot$ ist. In dieser Zusammensetzung wird sogar ein zusätzlicher männlicher Artgenosse toleriert, wenn er einige Tage lang Körperkontakt vermeidet. Auf generelle Dominanz weiblicher Desmana kann aus ihrer regelmäßigen Lokaldominanz im Bau geschlossen werden.

\section{Einleitung}

Der Russische Desman (Desmana moschata) (Abb. 1) ist ein urtümliches, wegen seiner weitgehenden Spezialisierung jedoch besonders gefährdetes Säugetier. Als Folge übermäßiger Bejagung zur Pelzgewinnung und progressiver Habitatzerstörung gingen seine Bestände um die letzte Jahrhundert- 
wende drastisch zurück. Weder das Jagdverbot aus dem Jahr 1920, noch die Aufnahme in das Rotbuch der UdSSR (Bannikov et al., 1978; Borodin et al., 1985), verbesserten die Überlebenschancen. Verursacht durch die politischen Umwälzungen, die zunehmenden ökonomischen Probleme und die dadurch ausgelöste Gesetzlosigkeit der letzten Jahre, verschlechterten sie sich weiterhin.

Durch die semiaquatile Lebensweise, die sich daraus ergebende, schwierige Freilandbeobachtung und die problemreiche Haltung basiert unser Wissen um das Sozialverhalten dieser gefährdeten Tierart großenteils auf Vermutungen. Daher wird in dieser Arbeit auch auf jene neuen Verhaltenseinzelheiten hingewiesen, die erst im Zuge der Bearbeitung der intraspezifischen Kommunikation offenbar wurden.

\section{Ökologie und kognitive Möglichkeiten der Desmaninae}

Im Gegensatz zum besser bekannten PyrenäenDesman Galemys pyrenaicus E. Geoffroy SaintHilaire, 1811, bevorzugt Desmana moschata Gewässer in breiten Flußtälern, die sich während der warmen Jahreszeit zu einer Kette lagunenartiger, strömungsloser und dadurch wenigstens temporär verschlammter Seen wandeln. Zur Zeit der Schneeschmelze steigt der Wasserstand, und die einsetzende Strömung bringt sauerstoffreiches Wasser und Nahrung (Insekten, Weichtiere und Fische) auch in die kleinsten, bis dahin voneinander isolierten Wasserbecken. Desmana moschata kommt zwar auch in ganzjährig langsam fließenden Gewässern vor, doch scheinen diese für ihn weniger attraktiv zu sein. Die Klarheit des Wassers ist für sein Vorkommen von geringer Bedeutung, denn auch - zumeist durch sympatrisch lebende Biber (Castor fiber Linnaeus, 1758) oder Bisamratten (Ondatra zibethicus Linnaeus, 1766) - getrübtes bzw. verschmutztes Wasser wird akzeptiert. Dies läßt annehmen, daß das akustische und das olfaktorische Kommunikationssystem im Wasser wichtiger sind als das optische. Zur Verifizierung dieser Hypothese ist es angezeigt, Verhaltensaspekte zuerst in möglichst naturgetreuen, künstlichen Anlagen zu studieren, bevor sie mit den - zwangsläufig schwierigeren - Freilandbeobachtungen korreliert werden können.

Welchen Stellenwert die anderen Sinnessysteme bei der intraspezifischen Kommunikation haben, bzw. ob und wie sie mit dem akustischen verschränkt sind, wurde noch nicht bearbeitet. Trotzdem sollen ihre relative Wertigkeit und ihre funktionelle Bedeutung (soweit bekannt) kurz erwähnt werden: Der Bulbus olfactorius gewährleistet einen guten Geruchssinn, die olfaktorischen Zentren des Gehirns sind aber gegenüber terrestrischen Talpiden reduziert (Stephan \& Bauchot, 1968; Stephan et al., 1991). Trotzdem dient der olfaktorische Sinn sowohl der Orientierung im Gelände als dem Sozialverhalten: Mittels der Faeces und der Sekrete der Moschusdrüse (Weber, 1904; Sokolov et al., 1977) werden sowohl über wie unter Wasser stark riechende Duftmarken abgesetzt. Es ist anzunehmen, daß diese chemischolfaktorische Orientierungshilfe in dem häufig trüben, moorigen oder schlammigen Wasser von kinästhetischen Fähigkeiten ergänzt wird. Wie bei allen Talpiden stehen für die haptische Orientierung zahlreiche Sinneskörperchen im Rüssel, gut innervierte Vibrissen, sowie Haarkämme an den Außenseiten der Hinterpfoten zur Verfügung (Stephan \& Bauchot, 1968). Hingegen ist das Sehvermögen schwach, gehaltene Tiere reagieren kaum auf eine (auch starke!) Lichtquelle oder auf die Annäherung einer menschlichen Hand auf wenige Zentimeter. Dies bestärkt die Vermutung, daß der chemisch-olfaktorische Sinneskomplex (Geruch und Geschmack) und der der akustische Sinn auch beim Sozialverhalten von Desmana moschata von überwiegender Bedeutung sind. Über eine in diesem Zusammenhang mögliche Bedeutung des Organum jacobsoni ist bis jetzt nichts bekannt.

\section{Material und Methoden}

Grundbedingungen zum Studium gehaltener Desmana moschata

Für eine erfolgreiche Haltung ist in erster Linie ein ausreichend großes Wasserbecken nötig. Da Kot und Harn auch im Wasser abgesetzt werden, müssen dessen Volumen entsprechend groß und die Filterung effizient sein.

Eine weitere Grundbedingung ist die Ermöglichung einer 


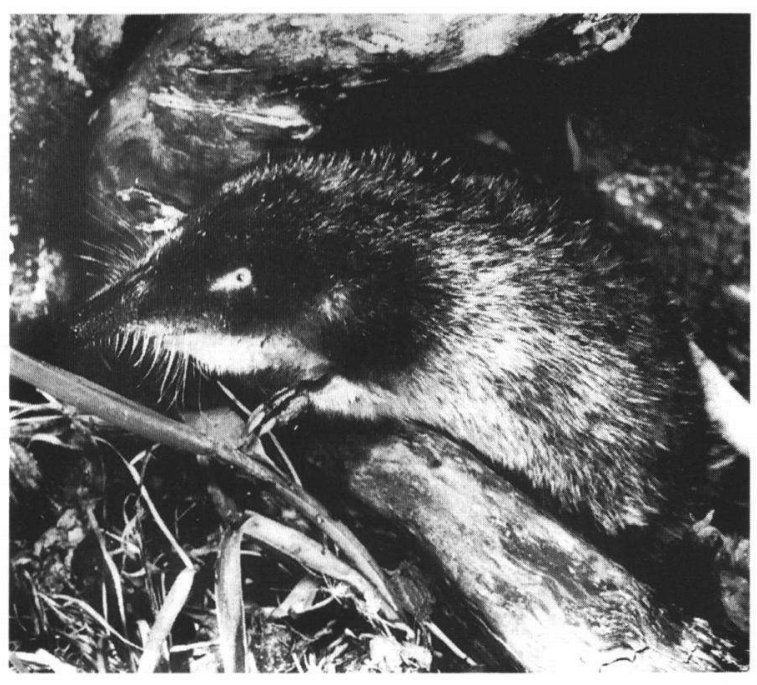

Abb. 1. Desmana moschata. Lebendaufnahmen sind außerordentlich rar (Photographie von P. Romanow).

artgerechten Fellpflege. In natürlichen Desman-Bauen ist die Länge der Schlupfröhren sehr unterschiedlich und hängt wohl von der Bodenbeschaffenheit ab. In Haltungssystemen dürfen sie jedoch nicht zu kurz sein. Ein freilebender Desman reinigt und trocknet sein Fell nach jedem Wasserkontakt in aller Ruhe durch gründliches Belecken, Schütteln und Kratzen. Diese Ruhe ist in den meisten Zoobetrieben nicht gegeben. Zudem haben mißlungene Haltungsversuche erwiesen, daß sich besonders bei ungenügender Filtrierung der zur Verfügung stehenden Wassermenge - in einem zu kurzen Gang sehr bald Futter- oder Pflanzenreste, Schlamm, Faeces, lose Teile der Gangwände etc. absetzen und die lebenswichtige Säuberung des Fells erschweren. Werden hingegen genügend lange, aus stabilem Material gefertigte Röhren geboten, vermindert sich diese Gefahr. Engstellen zum Abstreifen des Wassers, wie sie vom Schnabeltier (Ornithorhynchus anatinus (Shaw \& Nodder, 1799)) bekannt sind, benötigt der Desman nicht.

Für die Untersuchung der Lautgebungen des Desmans ist von ausschlaggebender Bedeutung, daß die Tiere nicht solitär gehalten werden. Nach unseren Erfahrungen können Einzelexemplare zwar jahrelang in Gefangenschaft leben, bleiben dabei aber zumeist stumm und verhalten sich somit - zumindest akustich - untypisch: Das Studium ihres Lautinventars ist bei Solitärhaltung praktisch unmöglich").

\section{Tiermaterial, Forschungslokalitäten und Methoden}

Die Untersuchungen wurden stufenweise in zwei Haltungssystemen und einem natürlichen Habitat durchgeführt:

-) Zusammenleben in Paaren oder Gruppen ist zwar bei Insectivoren selten, ist aber auch bei einem anderen semiaquatilen Talpiden, Condylura cristata (Linnaeus, 1758) schon festgestellt worden.
(1) Zwischen 1983 und 1986 konnten im Moskauer Zoo 5 Exemplare $\left(4 \sigma^{\circ} \sigma^{\circ}\right.$ und 1 \%) in wechselnder Zusammensetzung gehalten werden. Die Untersuchungsanlage sollte dem Studium der Haltungsfaktoren und der Biologie der Tiere, besonders aber der so oft vergeblich versuchten, regelmäßigen Zucht von Desmanen dienen. Nach Planung des Seniorautors (P.R.) wurden ein betoniertes Wasserbecken (Volumen etwa $40 \mathrm{~m}^{3}$ ) und drei etwa $40 \mathrm{~cm}$ unter dem Wasserspiegel beginnende, schräg nach oben führende Schlupfröhren (Durchmesser $10 \mathrm{~cm}$ ) angelegt. Sie mündeten jeweils in eine wagrecht liegende, rechteckige Nestkammer $(90 \times 40 \times 10 \mathrm{~cm})$. Etwa in deren Mitte befand sich eine kleinere rechteckige Schlafkammer $(25 \times 20 \times$ $10 \mathrm{~cm}$ ) mit 2 runden, diagonal angeordneten, seitlichen Einstiegslöchern (Durchmesser $10 \mathrm{~cm}$ ). Dadurch waren sowohl ein ungehindertes Betreten bzw. Verlassen der Schlafkammer, als auch eventuell nötige Fluchtwege um die Schlafkammer herum gewährleistet. Die Nestkammern waren durch einen abhebbaren Deckel von oben zu öffnen und geräumig genug, auch den Futternapf aufzunehmen. Diese Anlage erwies sich zwar nicht als ideal, entsprach aber insoweit den Lebensbedürfnissen der Tiere, daß sie darin immerhin 3 Jahre am Leben blieben.

(2) Zwischen 1989 und 1992 wurden in der Feldstation des Instituts für Tiermorphologie und Ökologie der Akademie der Wissenschaften der (ehemaligen) UdSSR in Cernogolovka ebenfalls 5 Exemplare ( $1 \sigma^{\circ}$ und $4 \%$ $\%$ ) gehalten und beobachtet. Als Wasserreservoir stand ein etwa $1000 \mathrm{~m}^{3}$ fassender, bis zu $2 \mathrm{~m}$ tiefer Teich zur Verfügung, im welchem die Fundamente der Beobachtungsstation standen (Abb. 2). Die Ufer und der Teichgrund waren naturbelassen und schlammig, sodaß die Tiere - zusätzlich zur täglichen Fütterung - zu jeder Tages- und Nachtzeit nach Nahrung suchen konnten. Zu diesem Zweck wurden Fische, Libellenlarven, Wasserinsekten etc. ausgesetzt. Von den Desmanen gegrabene Gänge in den Uferbänken wurden belassen und ständig kontrolliert. Mehrere Eingänge führten in den gefluteten Hauskeller und von dort in einen für Versuchszwecke variabel konzipierten Bau, der im Gegensatz zu der Anlage im Moskauer Zoo nicht wagrecht, sondern in mehreren Ebenen angelegt und in zahlenmäßig mehr, jedoch kleinere Kammern (etwa $30 \times 20 \times 10 \mathrm{~cm}$ ) gegliedert war. Zur Schaffung etwaiger Fluchtmöglichkeiten waren sie mit mehreren Ausgängen in die jeweilig angrenzenden Kammern versehen. Um lückenlose Lebendbeobachtungen im Bau zu ermöglichen, wurden die Vorderseiten sämtlicher Einzelkammern verglast.

Die Anlage war für die Haltung und Beobachtung von bis zu 20 Tieren konzipiert, wobei vor allem offene Fragen der Sinnesphysiologie und der Fortpflanzung geklärt werden sollten.

(3) Zwischen 1988 und 1992 wurden zahlreiche Freilandbeobachtungen im Oka-Reservat durchgeführt, 1990 auch in Anwesenheit des Zweitautors (W.P.). Derzeit umfaßt das 1935 gegründete, vom Staatlichen Ministerium für Naturschutz verwaltete Reservat 22.911 Hektar und enthält neben ausgedehnten Föhren- und eingestreuten Birkenwäldern zahlreiche sumpfige Niederungen mit flachen Seen (Abb. 3). Das Reservat liegt etwa $250 \mathrm{~km}$ südöstlich von Moskau bei dem Dorf Lakasch (Rjazan-Gebiet, Spasski-Bezirk) und wird von den Flüssen Oka 


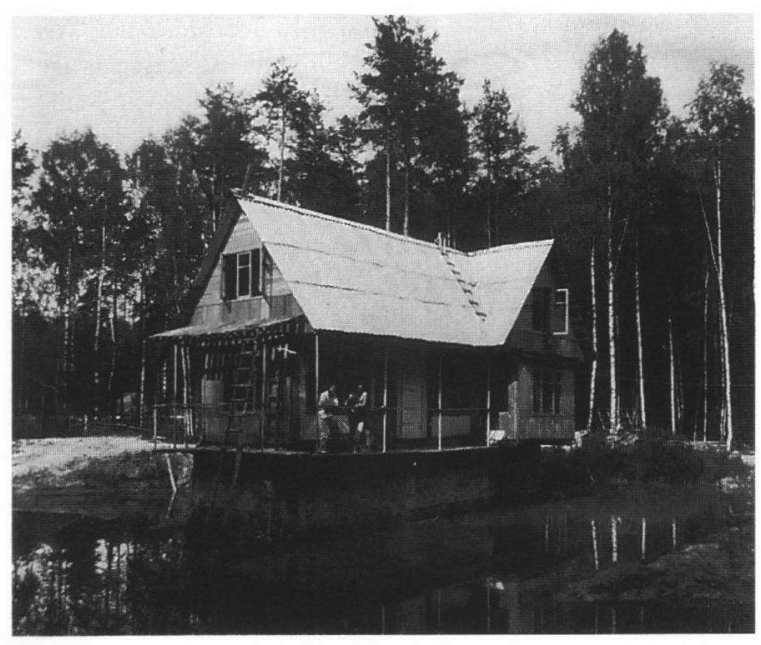

$A b b$. 2. Das in einen Teich gebaute Untersuchungshaus in Cernogolovka (Photographie von W. Poduschka).

und Pra entwässert. Zu Beginn des 20. Jahrhunderts war hier das größte Feuchtgebiet Europas: Damals bestanden 18\% des Areais aus Sumpf- und Wasserflächen. Heute sind diese als Folge ausgedehnter Trockenlegungen zum Zwecke landwirtschaftlicher Nutzung auf ein Fünftel reduziert. Während des alljährlichen Hochwassers im Frühling treten die Flüsse etwa 6 Wochen lang mehrere Meter hoch und in einer Breite von bis zu $30 \mathrm{~km}$ über die Ufer. Die Desmane überleben dies durch Zurückziehen auf die höchsten Erhebungen. Werden auch diese überflutet, beziehen die Tiere bis zum Sinken des Wasserspiegels verlassene Spechthöhlen in alten Bäumen oder sammeln Material für eine "Notunterkunft" in Astgabeln. Zumeist leben sie dort paarweise, doch wurden auch 3 oder 4 Individuen zusammen gefunden (Onufrienja \& Onufrienja, 1992). Über das Geschlechterverhältnis und die Verträglichkeit in solchen Gruppierungen ist nichts bekannt.

\section{Durchführung}

\section{Beobachtungen im Zoo von Moskau}

Bereits bei unseren Beobachtungen im Zoo drängte sich die Frage auf, ob Desmane tatsächlich dämmerungs- bzw. nachtaktiv sind, wie vielfach behauptet wurde: Da sie im Zoo keine natürlichen Futterquellen zur Verfügung hatten, mußten wir sie auch während des Tages füttern, was möglicherweise ein Eingriff in ihre natürliche Tagesrhythmik war. Ob die Konsumation dieses Futters die Ursache war, daß sie im Laufe des Tages - ausgenommen nachmittags - (insgesamt) etwa 1560 Minuten lang zur intensiven Fellpflege und/ oder zum Trinken den eigentlichen Bau verließen, war uns noch unbekannt: Im Zoo stiegen die Tiere dazu in einer Schlupfröhre bis zum Wasserspiegel ab, putzten sich dort und/oder tauchten auch kurz ins Wasser um zu trinken, verließen aber dabei nie die Röhre. Daß sie im Zoo tagsüber nicht das Wasser des Beckens tranken, war wohl durch die mangelnde Wasserqualität oder sonstige Störungen bedingt.

Immerhin ermöglichte uns die Anlage bereits 1986 zahlreiche Aufnahmen ihrer akustischen Signale mittels eines Tonbandgerätes (Reporter 3 UdSSR). Ein erstaunlich reichhaltiges, nach unserer damaligen Vermutung aber noch unvollständiges Lautrepertoire war dadurch erwiesen. Auf Zusammenhänge mancher Signale mit bestimmten Verhaltensweisen und somit auf ihre biologische Bedeutung konnte bereits geschlossen werden. Allerdings war in dieser Bearbeitungsphase noch unklar, ob die im Zoo gewonnenen Ergebnisse auch der natürlichen Verschränkung von Verhalten und Kommunikation im Freileben entsprachen: Wir mußten damit rechnen, daß sie durch das lärmende Zoomilieu oder durch Haltungsfehler modifiziert waren. Daher mußte unser nächster Schritt die Überprüfung und Deutung der Signalfunktionen in einem, dem natürlichen Biotop ähnlicheren Gehege sein. Dort erwies sich die genannte Befürchtung als richtig.

\section{Haltungs- und Teil-Freilanduntersuchungen in Cernogolovka}

In dieser Feldstation der Russischen Akademia Nauk konnten aufgrund der größeren und bedeutend naturnäher konzipierten Anlagen wesentlich differenziertere Sozialisierungssituationen und -variationen geprüft werden. Allein mit der Lautkommunikation haben wir uns rund $400 \mathrm{Be}-$ obachtungsstunden lang beschäftigt, wobei technische Gefahrenquellen wie etwa Übersteuerungen oder Fremdgeräusche durch Wiederholungen und Einstellungsänderungen tunlichst vermieden wurden. 


\section{Freilanduntersuchungen im Oka-Reservat}

Synchron mit den Arbeiten in Cernogolovka wurden zur oben postulierten Kontrolle der Ergebnisse auch hier die Freilandbeobachtungen fortgesetzt. Bei langsamem Abgehen jener Uferränder, die nicht aufgrund meterdicker Wasserund Sumpfpflanzenbestände unzugänglich waren (vergl. Abb. 3), konnte man die Tiere außerhalb ihrer Baue aus verschiedenen Entfernungen mehr oder weniger gut und lange beobachten und natürlich auch hören. Es war aber ein durch ganz ungewöhnliche klimatische Verhältnisse begünstigter Sonderfall, daß einer der Autoren (P.R.) unmittelbar neben einem in der Uferböschung befindlichen Bau stehend - im Oktober 1988 freilebende Desmane etwa drei Stunden lang (12 Uhr mittags bis ca. $15 \mathrm{Uhr}$ ) aus nächster Nähe beobachten konnte: Noch vor dem ersten Schneefall hatte Frost eingesetzt, so daß die temporären "Seebecken" von einer durchsichtigen Eisschicht überzogen waren. Damit war für die schwachsichtigen Desmane (vergl. Kapitel "Ökologie und kognitive Möglichkeiten...") offensichtlich sowohl das optische wie das olfaktorische Erkennen einer Gefahr stark behindert oder gar ausgeschlossen.

Durch die dünne Eisdecke, welche die akustischen Signale passieren ließ, waren die Aktivitäten mehrerer fouragierender Desmane gut zu beobachten. Da die Tiere während des Schwimmens im natürlichen Gewässer kaum voneinander unterschieden werden können, war ihre Anzahl schwer festzustellen und kann nur grob mit 2-4 angegeben werden. Sie kamen wiederholt aus der unter dem Wasserspiegel mündenden Schlupfröhre heraus und kehrten nach kurzer Zeit mit Beute bzw. Nestmaterial(?) im Maul zurück. Dabei waren - in vergleichbaren Verhaltens-Situationen - dieselben Signale zu hören wie bei den gehaltenen Tieren im Moskauer Zoo und in der Station Cernogolovka.

Anläßlich dieser Beobachtung ist es angebracht, über die Tagesrhythmik von Desmana moschata zu referieren: Erst im Oka-Reservat konnten wir feststellen, daß man bestenfalls von einer bedingten bzw. teilweisen Dämmerungs- oder Nachtaktivität sprechen kann. Sie ist im Freileben ungleich aufgelockerter als unter Haltungsbedin-

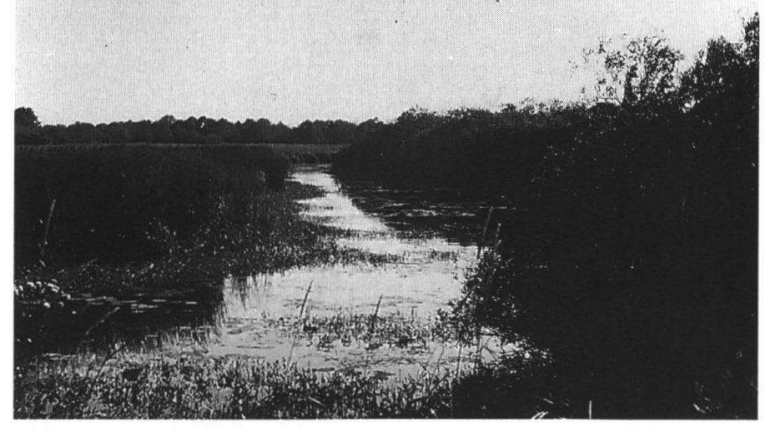

$A b b$. 3. Lagunenartige Flußbecken im Sommer. Die Ufer sind dicht bewachsen, haben aber vielfach steile, gut $1 \mathrm{~m}$ hohe Ränder, so daß die nur vom Wasser zugänglichen Wohnhöhlen der Desmane oberhalb der Wasserlinie liegen. (Photographie von W. Poduschka.)

gungen, denn die Tiere kommen auch untertags, ohne besondere Zeitpräferenz, häufig zum Vorschein um Nahrung zu suchen bzw. Nestmaterial(?) einzutragen, wobei man einen ungefähren Mittelwert von insgesamt(!) etwa 3 Stunden angeben kann. Besonders im Spätherbst nimmt die Tagesaktivität $z u$, da die Tiere neue Höhlen graben, um im Winter die Gewässer besser nützen zu können. $\mathrm{Ob}$ dies aber vorbereitete, temporär leerstehende Ausweichquartiere sind, die gegebenenfalls aufgrund unvorhersehbarer äußerer Umstände (etwa große Kälte, dadurch dickere Eisdecke und Fehlen von ufernahen Lufträumen zum Atmen, etc.) oder einfach durch Populationsdruck aufgrund des sommerlichen Nachwuchses entstehen, konnten wir nicht erkennen. In früheren Jahren war es gebräuchlich, die Populationszahlen anhand der ausgemachten Einschlupfröhren anzugeben, doch hat man diese in jeder Hinsicht irrelevante, jedoch jahrzehntelang mit denselben Zahlen weiterkolportierte Methode längst aufgegeben.

Leider beendeten die politische Entwicklung in Rußland und die steigenden ökonomischen Schwierigkeiten im Jahre 1992 die erhoffte Fortführung der Forschungen in Cernogolovka und verhinderten vorerst auch die Auswertung der bisherigen Ergebnisse vor dem Februar 1993. Erst dann konnten im Institut für Schallforschung in Wien die besten Tonbandaufnahmen vom Original- auf ein Arbeitsband kopiert und von dort auf etwa 150 Einzelblätter überspielt werden. Daraus wurden die besten Aufzeichnungen ausgewählt, gemeinsam ausgewertet 


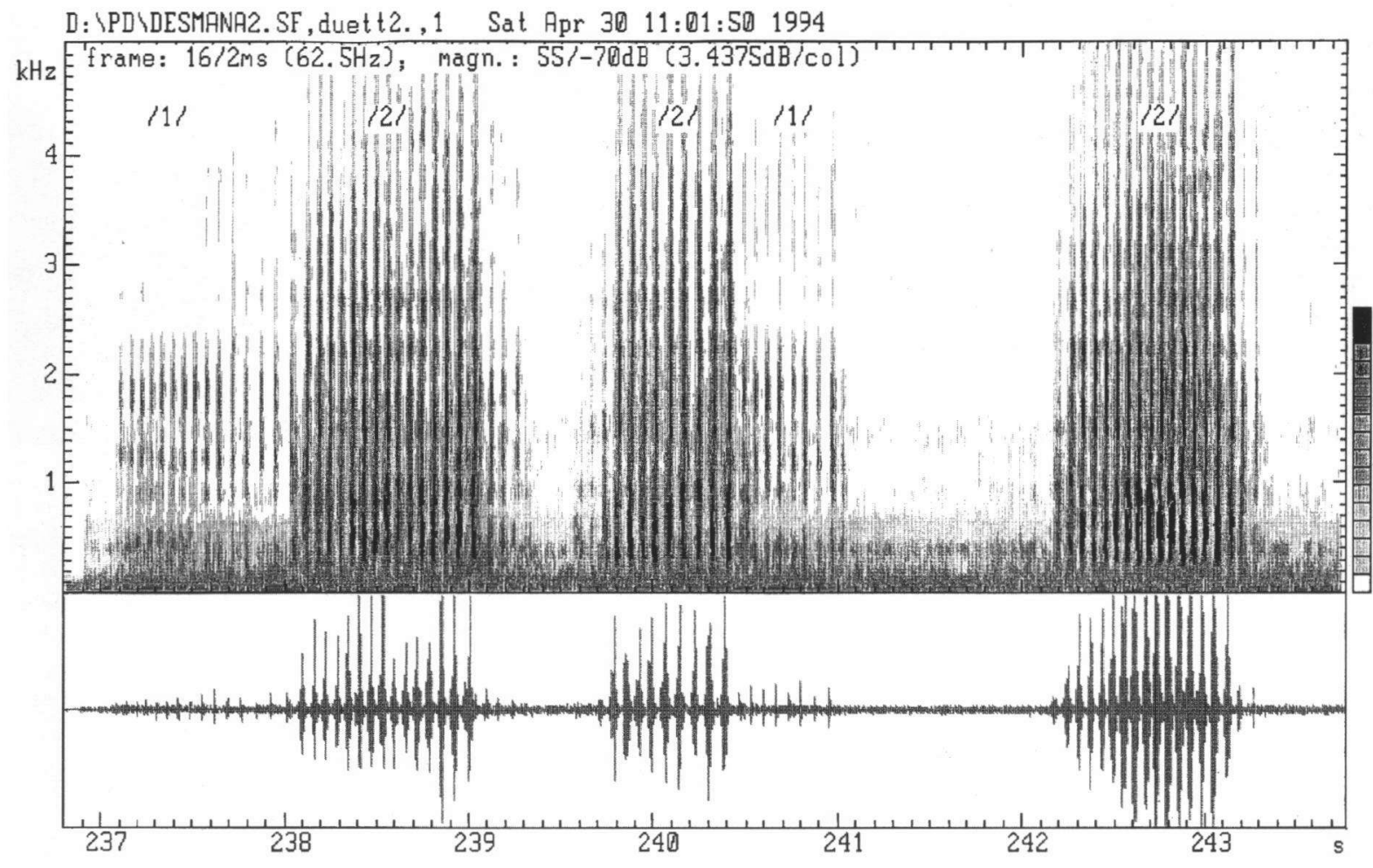

$A b b$. 4a. Sonagramm des "Knattern". Responsorialkommunikation zweier $D$. moschata, /1/ entfernt, $/ 2 /$ näher zum Mikrophon. Frequenzschwerpunkt zwischen 300 und $600 \mathrm{~Hz}$, obere Grenzfrequenz ca. $5000 \mathrm{~Hz}$. Wiederholungsrate der Einzelpulse /2/: $58 \mathrm{~ms}$, /1/ : 61,5 ms. Gegen Ende der einzelnen Pulsketten nimmt die Regelmäßigkeit ab. (Sonagramm und Text von W. Deutsch.)

und anhand der Originalprotokolle mit den in allen Untersuchungsphasen beobachteten, synchronen Verhaltensweisen korreliert. Aus Platzgründen können hier leider nur die wichtigsten und eindeutigen Signale veröffentlicht werden. Zahlreiche weitere, häufig mehrdeutige Vokalisationen und ihre Kombinationen müssen künftigen Bearbeitungen vorbehalten bleiben.

Die technischen Details (wie Signallängen oder -strukturen, Frequenzhöhen, Harmonische, etc.) sind jeweils aus den Legenden der Sonagramme zu entnehmen.

\section{Ergebnisse und Diskussion}

Über Lautäußerungen des Russischen Desmans wurde bereits mehrfach, jedoch wenig übereinstimmend berichtet: Barabash-Nikiforow (1975) erwähnt eine Art von Knurren, Pfiffe, zirpende oder stöhnende Laute des Männchens beim Treiben des Weibchens, sowie zirpende Lockrufe des letzteren. Das von diesem Autor erwähnte “unwillige krr-krr-krrr" (: 71) könnte mit dem von uns "Knattern" genannten Signal identisch sein. Nach Sokolov et al. (1976) lassen aggressive Individuen einen "Knall"(?) hören"*). Leider konnten wir die meisten der von diesen Autoren genannten Signale nicht hören bzw. deren Beschreibungen nicht verstehen und daher nicht mit unseren Verhaltensbeobachtungen korrelieren. Wir müssen uns somit auf unsere akustischen Wahrnehmungen beschränken, die aber nicht nur das in der Folge erwähnte reichhaltige Lautinventar, sondern auch eine Anzahl variabeler Signalkombinationen ergaben.

**) Infolge der individuell verschiedenen Erlebnisqualität akustischer Perzeption ist es allerdings problematisch, Tiersignale mit Worten zu beschreiben. Dies macht vor allem Übersetzungen in andere Sprachen fast unmöglich. 

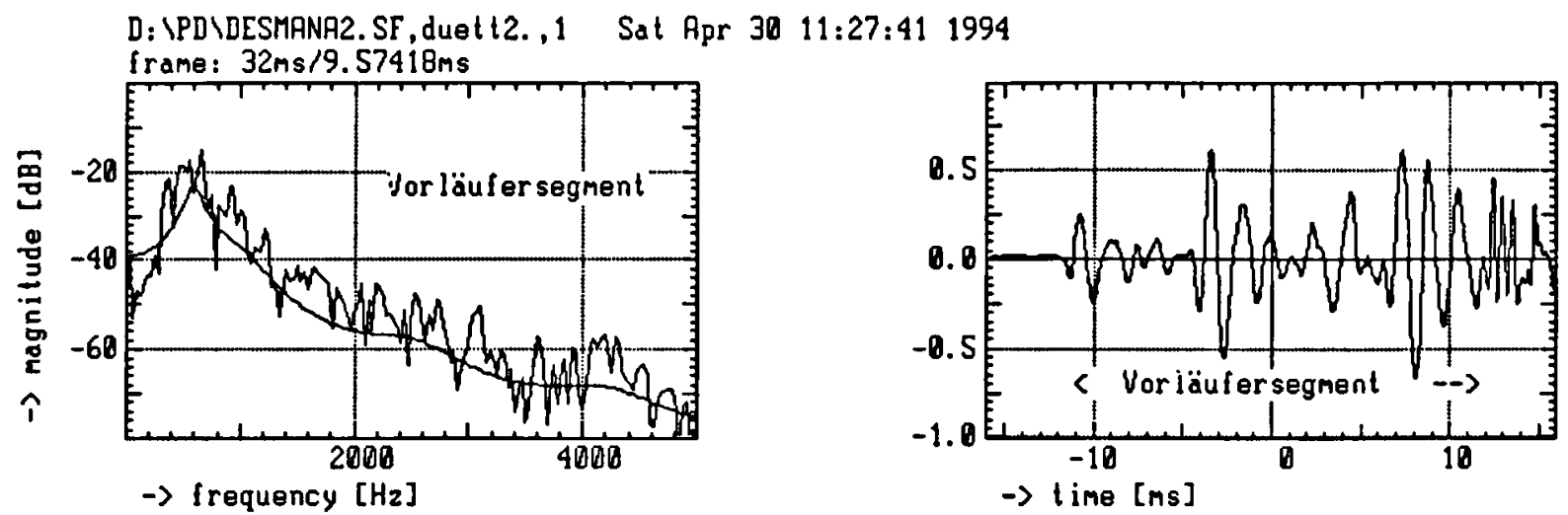

D: IPD\DESMANA2. SF, duet 12. ,1 Sat Apr 30 11:31:49 1994
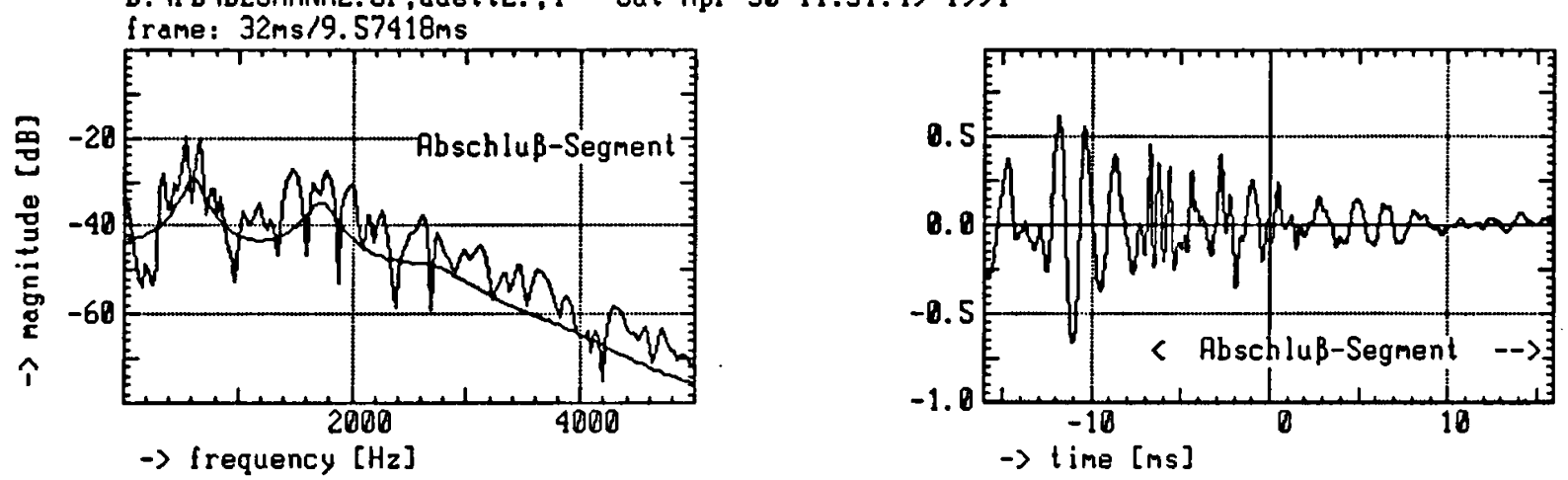

$A b b .4 b$. Sektion. Links: Amplitudenspektrum, rechts: Wellenform. Die Einzelpulse bestehen aus 2 Teilen, einem "Vorläufer"-Segment mit dem stationären Frequenzschwerpunkt bei ca. $600 \mathrm{~Hz}$ und der Dauer von ungefähr $20 \mathrm{~ms}$, gefolgt von dem zweiten Teil (AbschlußSegment), ebenfalls mit einer Dauer von ca. $20 \mathrm{~ms}$, aber mit zusätzlich höheren Frequenzanteilen. (Sonagramm und Text von W. Deutsch.)

\section{Knattern (Abb. $4 a \& b)$}

Das häufigste und auffälligste Signal des Russischen Desmans ist eine Art von "Rattern", "Trommeln" oder "Knattern". Da die beiden erstgenannten Ausdrücke in der Zoologie zur Charakterisierung akustischer Phänomene von diversen Autoren seit langem gebraucht werden ("Trommeln" bei Piciformes und Lagomorpha, "Rattern" bei Meeresbewohnern und diversen Säugern, vergl. Seite 50), bevorzugen wir den frequenzbezüglich neutralen Ausdruck "Knattern".

Die in der älteren russischen Literatur dafür gebräuchliche, phonetisch aus dem Deutschen übernommenen Bezeichnung "Triller" muß aufgrund unserer Sonagramme berichtigt werden. Diese Wortwahl ist falsch: Unter einem Triller versteht man den wiederholten, mehr oder weniger schnellen Wechsel eines
Tones mit seiner Obersekunde bzw. seine rhythmische Unterbrechung durch größere Intervalle. Das Sonagramm (Abb. 4a) zeigt aber, daß dies nicht der Fall ist: Die in schneller Folge aneinandergereihten Einzelsignale zeigen keine Frequenzunterschiede.

Diese Laute sind besonders häufig in der Dämmerung zu hören. Die Lautstärke hängt vom Erregungszustand der Tiere ab. Nach unserer Schätzung dürfte ihre Reichweite für das menschliche Ohr (über Wasser) etwa bei $40 \mathrm{~m}$ liegen. Ob dies mit der als gut anzunehmenden Hörkapazität der Desmane übereinstimmt, können wir nicht schlüssig beantworten. Da das Knattern bei Anwesenheit eines oder mehrerer Artgenossen fast immer beantwortet wird, ist es grundsätzlich als Stimmfühlungslaut $\mathrm{zu}$ werten. Dies geht auch daraus hervor, daß es von einem solitär lebenden Tier sowohl in Freiheit als auch im Haltungssystem 
kaum emittiert wird. Wird ein Pärchen in einem zu kleinen Gehege gehalten, in welchem sich die Tiere kaum voneinander entfernen können, ist das Signal anfangs zwar häufig, wird aber - wohl infolge eines Gewöhnungseffektes oder weil es kommunikativ sinnlos bleibt - zunehmend seltener. Bei Gruppenhaltung hingegen bleibt die Häufigkeit erhalten. Die Lautstärke variiert besonders stark bei Responsorial"duetten" (Abb. 4a).

Sowohl die Beobachtungen in freier Natur als auch bei gehaltenen Tieren erweisen das Knattern als multifunktionell. Wohl ist es generell ein Kontaktsignal, seine Bedeutungsskala reicht aber von demonstrativer Dominanz bis zur dazu konträren Submission. Dominanz mit Akzeptierung des Partners wird z.B. angezeigt durch lautes Knattern von Seiten des einen Exemplares mit eventuellem, aber nicht obligatem Zeigen der Zähne, nicht aber durch Emission des Abwehrsignals. Die Submission wird durch das korrelierte akustische und motorische Verhalten des anderen Tieres kenntlich, das sich - bedeutend leiser knatternd - wie zögernd an das dominante Tier heranschiebt und unter noch anhaltendem leisem Knattern in dessen Nähe verbleibt (cf. Abb. 4).

Lautstärke und Dauer der Signalfolgen hängen von der jeweiligen Erregungsintensität und/oder von der sich ändernden Individualdistanz $a b$. Dies wird besonders deutlich, wenn sich ein Exemplar schwimmend dem Eingang eines Baues nähert: Das Knattern beginnt gewöhnlich in etwa $3 \mathrm{~m}$ Entfernung zum Partner und endet, graduell leiser werdend, erst bei etwa $20 \mathrm{~cm}$. In dieser Situation hat das Signal offenbar eine Art nicht submissiver "Anmelde"-Funktion, andernfalls müßte das Signal $\mathrm{Abb} .5$ eingesetzt werden. $\mathrm{Ob}$ es vom Partner individuell erkannt werden kann, ist ebensowenig bekannt, wie die theoretische Möglichkeit, daß es beim Anschwimmen eines Zieles als Echo-Peilung eingesetzt wird, vergleichbar den Ratterlauten von Fledermäusen (Möhres, 1952), den "clicks" von Soriciden (Gould, 1969) und Tenreciden (Gould, 1965), dem "rusty-hinge-creaking" von Meeressäugern (Schevill \& Lawrence, 1953) und von Erinaceinen (Poduschka, 1968; 1969), aber auch vom Iberischen Desman Galemys pyrenaicus durch die "tambourinage", einem rhythmischen Schlagen der Vorderpfoten auf die Wasserfläche bei Annäherung an ein Hindernis (Richard, 1986).

Lebt ein Pärchen schon längere Zeit zusammen, dann bleibt das Signal zumeist verhältnismäßig leise, ist dabei aber von beiden Tieren zu hören. Die Erregung ist demnach gering und das gedämpft klingende Signal ist wohl als Ausdruck von Vertrautheit mit dem Sozialpartner zu werten.

In einem Haltungssystem ist das Knattern besonders dann oft und stark zu hören, wenn ein weiteres Exemplar mit einem oder zwei bereits aneinander gewöhnten vergesellschaftet wird. Dies weist darauf hin, daß die - unter diesen Umständen besonders erregten - eingesessenen Tiere ihre Signale wohl auch als Drohlaut einsetzen. Die Intensität flaut erst nach etwa 3 Tagen $a b$, sofern sich der Neuankömmling ruhig verhält, Körperkontakte durch Zurückziehen in Blindgänge vermeidet und so einen steigenden Duldungseffekt erreicht. Man kann dies als Abbau der Territorialdominanz der residenten Exemplare deuten, wobei - nach unserer Erfahrung - immer das $\subsetneq$ die Alpha-Position innehat. Dafür spricht, daß das Knattern eines zum Höhleneingang strebenden Männchens unmittelbar vor dem Einschlüpfen in die Röhre regelmäßig in submissives "Piepsen" übergeht. Die Bedeutung des Knatterns in weiteren Situationen ist schwer $\mathrm{zu}$ interpretieren.

\section{Beschwichtigungs- bzw. Demutsignal (Abb. 5)}

Grundsätzlich scheint dieses "Piepsen" ein Erkennungslaut zu sein; ob es aber auch zur Selbstpräsentation oder als Beschwichtigungssignal gegenüber dominanten Artgenossen dient, ist noch fraglich. Besonders häufig ist es bei Annäherung einer Desmana an eine Nestkammer zu hören. Daher ist anzunehmen, daß es auch als Beruhigungs- oder Submissivlaut dient. Wir haben jedenfalls beobachtet, daß meist jede Aggressivität des dominanten Tieres unterbleibt, wenn ein anderes die kritische Distanz piepsend unterschreitet. 


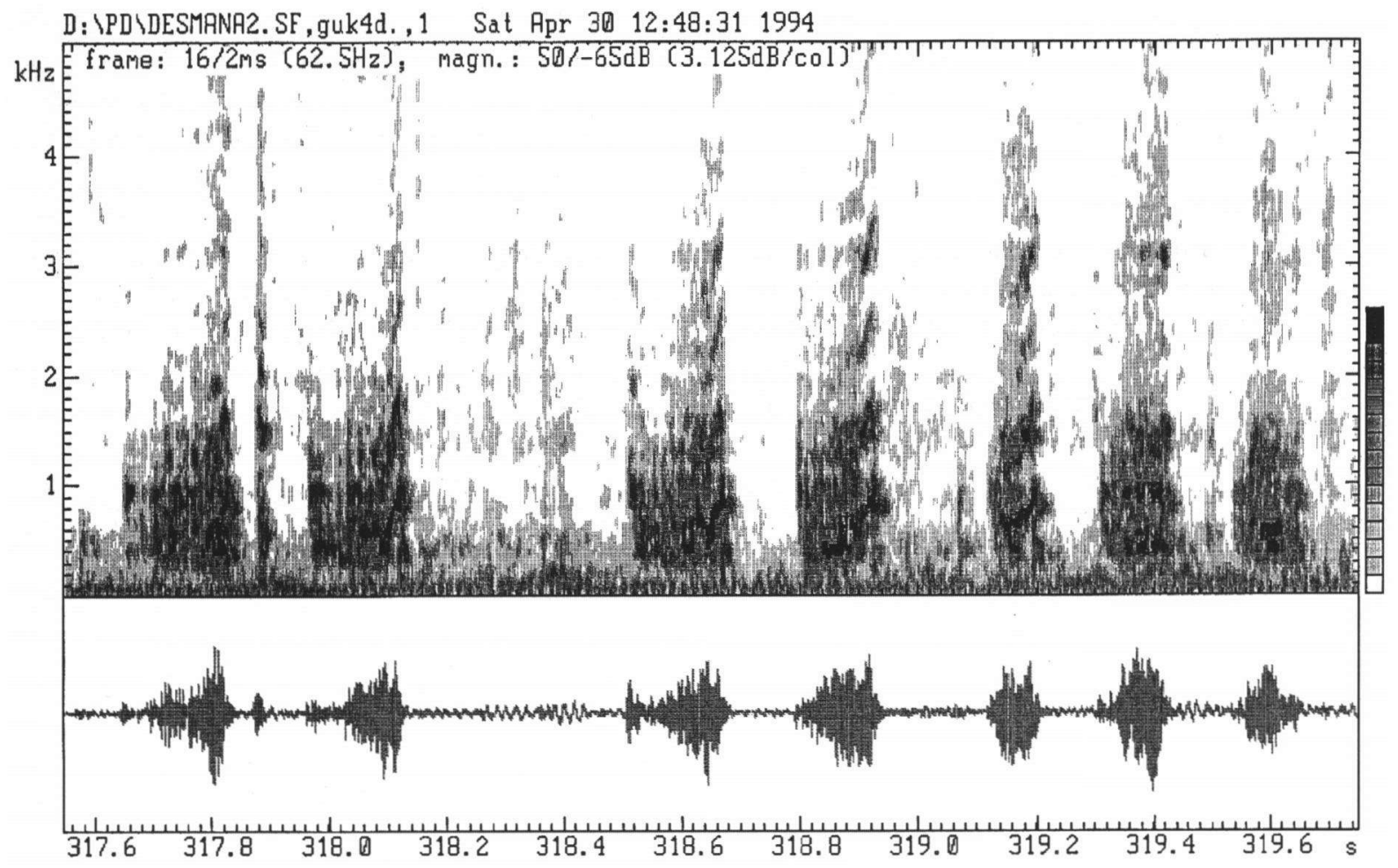

Abb. 5. "Demut". Stimmlaut-Sequenz. Quelle vermutlich Glottisaktivität. Harmonischer Klang mit Energieschwerpunkt auf der Grundfrequenz. Häufig u-förmiger Tonhöhenverlauf; Variationsbreite 750-1100 Hz, Mittelwert bei $900 \mathrm{~Hz}$. (Sonagramm und Text von W. Deutsch.)

\section{Positives Direktkontakt-Signal (Abb. 6)}

Dieses weder aggressiv noch submissiv klingende Signal, das beim Partner keine sicht- oder hörbare Reaktion auslöst, drückt wohl Behaglichkeit und Sicherheit im jeweiligen Sozialverband aus. In gleichwohl sehr vorsichtiger, vager Anlehnung an Lautkommunikationen anderer Säuger in entsprechender Situation könnte man es vielleicht für ein positives Nahkontakt-Signal halten.

\section{Abwehr-bzw. Drohsignal (Abb. 7)}

Diese besonders erregt klingenden, kreischenden Signale sind als Droh- bzw. Warnlaute anzusehen. Sie werden stets bei plötzlichem Direktkontakt und fast immer bei gegenseitiger Annäherung zweier Desmane hörbar, von denen ein Tier aus meist unbestimmbaren Gründen einen Kontakt vermei- den will. Kommt es zu einem Kampf, werden diese Signale sehr laut. Sie werden bei offenem Maul ausgestoßen (Beißintention, Zeigen der Zähne). Gleichzeitig werden mit den Vorderbeinen agonistische Intentionsbewegungen (Kratzen, Stoßen) ausgeführt.

Gewöhnlich werden diese Droh- oder Warnsignale in etwa 1,5 m Distanz begonnen, was vielleicht dem Maximum ihrer individuellen "Erkennbarkeit" entspricht. Handelt es sich um einander fremde Individuen und wird die individuell geschlechtsgebunden oder situationsbedingt unterschiedliche kritische Distanz unterschritten, erfolgt in der Regel eine heftige Attacke durch eines der Tiere. Kommt es zum Körperkontakt, sind schwere Bißverletzungen nicht selten; doch stoßen sich die Opponenten in den meisten Fällen mit den Vorderpfoten voneinander $a b$. Sehr oft genügen aber die hier demonstrierten Warnlaute zur Einhaltung der Individualdistanz bzw. zur Vergrößerung 


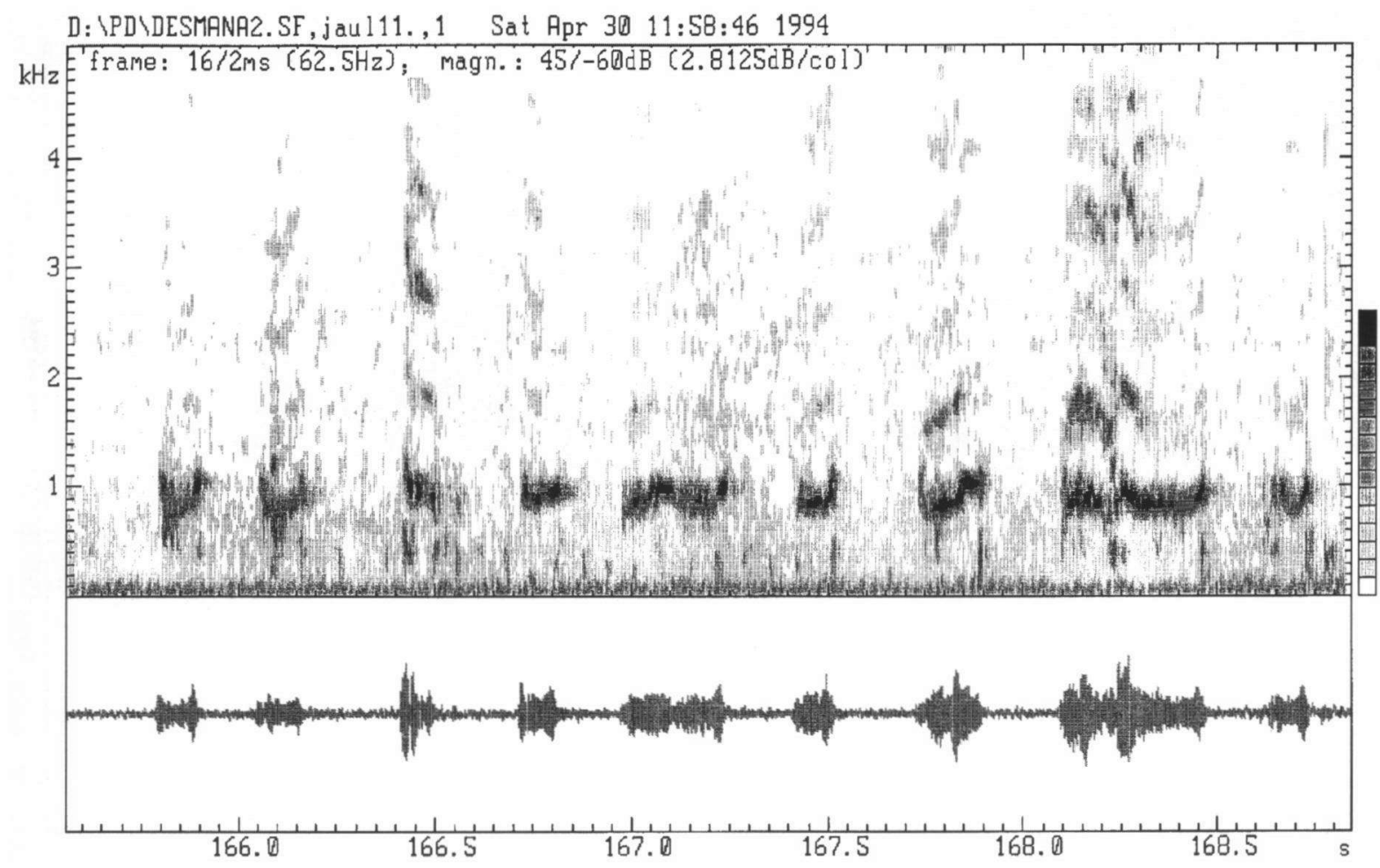

$A b b$. 6. Positiver Direktkontakt. Ruffolge, bestehend aus stimmhaften Einzellauten mit zumeist ansteigender Tonhöhe. Teilweise harmonische Teilstruktur. Ausgangsfrequenz: etwa $300 \mathrm{~Hz}$, Terminalfrequenz ca. $1100 \mathrm{~Hz}$ bei annähernd linearem Anstieg in den letzten 30-40 ms des Rufes. Der Einzelruf entwickelt sich häufig aus einem auf S. 48-50 gezeigten und beschriebenen Knattergeräusch. (Sonagramm und Text von W. Deutsch.)

des Abstandes; manchmal lösen sie auch eine spontane Fluchtreaktion eines der Opponenten aus.

\section{Verhaltensbezogene Schlußfolgerungen}

Zum Sozialverhalten kann generell gesagt werden, $\mathrm{da}$ weibliche Desmane initiativer und aggressiver sind als männliche, und zwar besonders gegen Geschlechtsgenossinnen, die häufig angegriffen und sogar getöten werden. $\mathrm{Ob}$ dabei Territoriumsbesitz oder eine bestimmte Östrusphase einen Einfluß auf den Ausgang des Kampfes haben mögen, ist noch nicht erforscht. Leider kann man im Freileben immature Weibchen von geschlechtsreifen nicht unterscheiden, sobald der Größenunterschied verschwunden ist. Nach unseren Erfahrungen mit gehaltenen Weibchen(!) tritt die Geschlechtsreife erst mit etwa 4 Monaten ein.
Sicherlich erkennen adulte Weibchen dies olfaktorisch, ob aber der eigene weibliche Nachwuchs eventuell erst $\mathrm{ab}$ der nächsten erfolgreichen $\mathrm{Be}-$ fruchtung angegriffen und vertrieben wird, oder $o b$ es darüber hinaus irgendwelche hemmende (olfaktorische, ethologische??) Barrieren gibt, ist noch nicht bekannt.

Abgesehen von der Paarungszeit, sind die Männchen gegeneinander grundsätzlich friedlich: Wie wir bei der Vergesellschaftung der 4 Männchen mit dem einzigen Weibchen in Cernogolovka feststellen konnten, gab es keinerlei Schwierigkeiten, was anzeigt, daß Russische Desmane grundsätzlich nicht in Familien, sondern in nicht unbedingt verwandten Gruppen leben. Bezüglich der Männchen wurde dies schon in der älteren russischen Desmanliteratur erwähnt, doch ist schwer $\mathrm{zu}$ eruieren, auf welchen Autor es zurückgeht.

Wir möchten betonen, daß die Signale des 


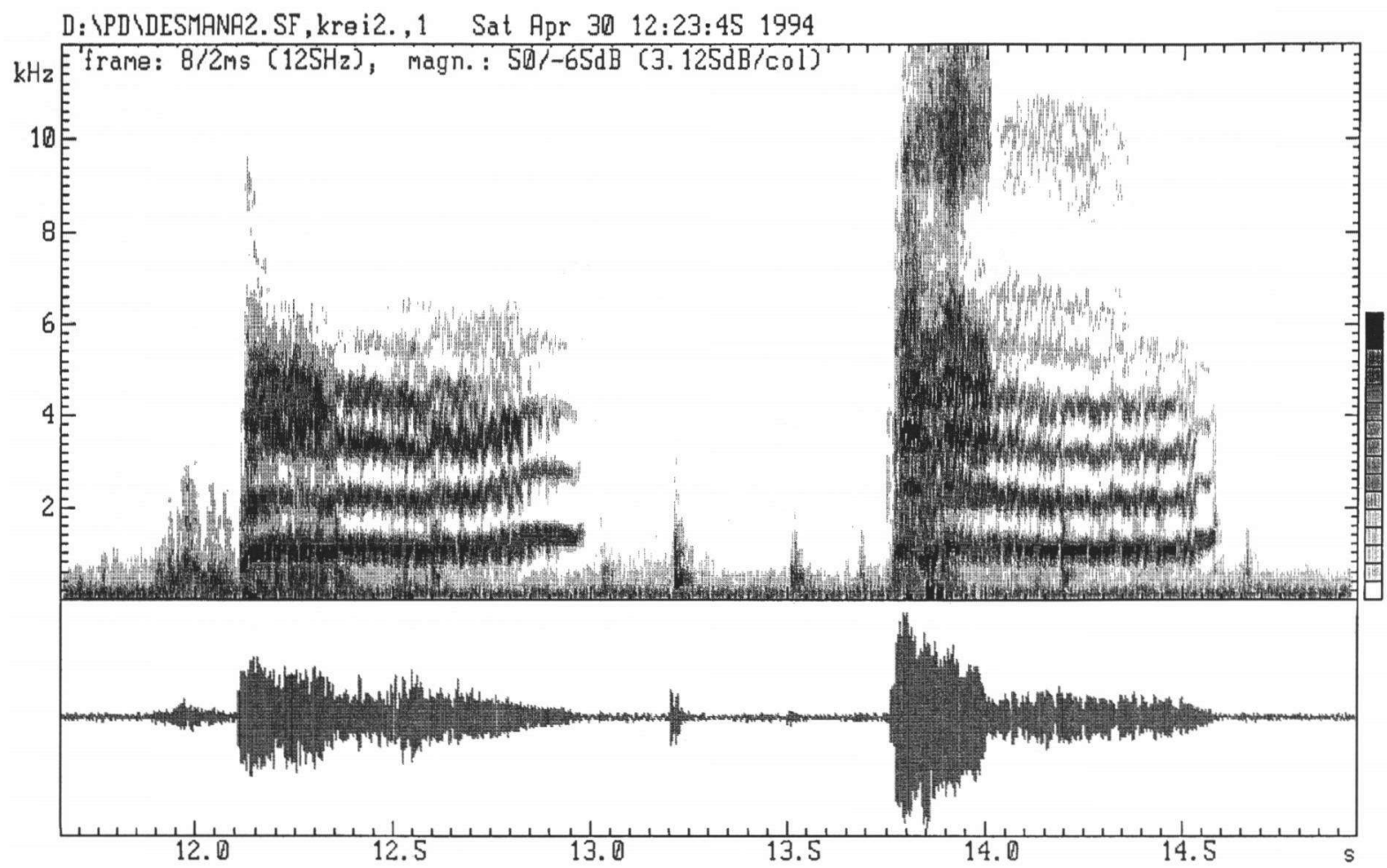

$A b b$. 7. Defensivsignal. Langgezogener Stimmlaut, Quelle wahrscheinlich Glottisaktivität, bestehend aus 2 Teilen: Das erste Drittel enthält Stimm- und Geräuschanteile, letztere mit starken Energieanteilen bis $12.000 \mathrm{~Hz}$. Die Stimmgrundfrequenz liegt bei ca. $1060 \mathrm{~Hz}$ mit geringer Variation nach oben in der Terminalphase des Rufes. Der Klang hat 4 bis 5 harmonische Teiltöne, die in den beiden letzten Rufdritteln ohne Geräuschanteile produziert werden. (Sonagramm und Text von W. Deutsch.)

Desmans außerordentlich modulations- und kombinationsfähig sind. Zusätzlich ergibt sich aufgrund ihrer Multifunktionalität und ihrer Intentionsunterschiede ein so reichhaltiges, mit bestimmten Verhaltensweisen korreliertes Lautinventar, daß die einzelnen Signaltypen nur schwer eindeutig kategorisiert werden können.

Nach unseren Befunden sind verschiedene Lautäußerungen für die Rangordnung innerhalb einer etablierten Gruppe wichtig. Sie spiegeln aber auch die jeweilige eigene Stimmung wider. Zeigt der Kontaktparner keine abweisende Haltung bzw. Lautgebung, so kommt es in der Regel bei beiden Tieren zu Behaglichkeitssignalen (Abb. 6). Daß Desmane Körperkontakt dulden und somit zumindest im gemeinsamen Bau - durchaus keine Solitärtiere sind, ergibt sich daraus, daß sie sich in einem Haltungssystem im Wachzustand zwar zumeist in verschiedenen Schlafkammern oder im trockenen Teil der Schlupfröhre aufhalten, jedoch zum Schlafen regelmäßig nur in einer Kammer, wie eng diese auch sein mag, zusammendrängen: 4-5 Exemplare in einer der oben beschriebenen Schlafkammern von $25 \times 20 \times 10 \mathrm{~cm}$ ist keine Seltenheit. Dabei ist natürlich infolge der notorischen Unverträglichkeit adulter Weibchen Grundbedingung, daß sich in solchen Schlafgemeinschaften nur ein solches befindet. In diesem Zusammenhang ergibt sich noch die bis jetzt unbeantwortbare physiologische Frage, ob nicht die individuelle Wärmeregulation der Desmane (wenigstens während ihres Ruheschlafes) vielleicht nur partiell homoiotherm ist.

\section{Danksagung}

Wir danken Akademiker W.E. Sokolov für die Ermöglichung der Arbeiten in der Feldstation Cernogolovka, sowie für die zweimalige Einladung des Zweitautors (W.P.) zum Studien- 
aufenthalt in Moskau (1983) und in Cernogolovka (1990). Für die Erstattung der Reisekosten sind wir der Österreichischen Akademie der Wissenschaften (Auslandsreferat) sehr verpflichtet. Die Stiftung Volkswagenwerk ermöglichte großzügig sowohl die jahrelange Grundlagen- wie auch Spezialforschung an Insektivoren und ihre Zucht. Dem Direktor des Moskauer Zoos, Dr. Wladimir Spitzin, danken wir ebenso wie dem Direktor des Oka-Reservats, Dr. Svjatoslav Priklonski und unserem Kollegen A.S. Onufrienja für seine fachkundige Beratung und Mitarbeit im Freigelände. Auch allen Freunden, die bei der Errichtung des Untersuchungshauses im nachmaligen Desmanteich in Cernogolovka so selbstlos geholfen haben, möchten wir unseren herzlichen Dank ausdrücken. Herm Univ. Prof. emer. Friedrich Schaller (Universität Wien) danken wir herzlichst für die Durchsicht des Mechanoskripts, sowie Herrn Univ. Prof. emer. Dr. Dr. h.c. Dietrich Starck (Universität Frankfurt a. M.) für die vieljährige Hilfe in mannigfacher Hinsicht.

\section{Literatur}

Bannikov, A.G., et al., 1978. Krasnaya kniga SSSR. Redkie i nakhodyashchiesya pod ugrozol ischesnoveniya vidy zhivotnykh i rastenil, chast' 1. Mlekopitayushchie (Red data book of USSR, part 1. Mammals): 13-14 (Lesnaya promyshlennost, Moskva).

Barabash-Nikiforow, I.I., 1975. Die Desmane. Familie Desmanidae (Insectivora). Die Neue Brehm-Bücherei, 474: 1-100 (A. Ziemsen Verlag, Wittenberg Lutherstadt).

Borodin, A.M., A.G. Bannikov \& V.E. Sokolov, 1985. Krasnaya kniga SSSR (2. Aufl.), 1: 12-13 (Lesnaya promyshlennost, Moskva).

Gould, E., 1965. Evidence for echolocation for the Tenrecinae of Madagascar. Proc. amer. phil. Soc., 109: 362-360.

Gould, E., 1969. Communication in three genera of shrews (Soricidae): Suncus, Blarina \& Cryptotis. Communs. behav. Biol., (A) 5: 11-31.
Möhres, F.P., 1952. Die Ultraschall-Orientierung bei Fledermäusen. Naturwissenschaften, 39: 273-279.

Onufrienja, A.S. \& M.V. Onufrienja, 1992. Desman in Oka State Reserve (Russia). Proceedings of the Meeting on the Pyrenean Desman, Lisboa: 81-83 (Servicio nac. de Parques \& Museu nac. Hist. nat., Lisboa).

Poduschka, W., 1968. Über die Wahrnehmung von Ultraschall beim Igel, Erinaceus europaeus roumanicus. $Z$. vergl. Physiol., 61: 420-426.

Poduschka, W., 1969. Ergänzungen zum Wissen über Erinaceus e. roumanicus und kritische Überlegungen zur bisherigen Literatur über europäische Igel. Z. Tierpsychol., 26: 761-804.

Richard, B., 1986. Le Desman des Pyrénées. Un mammifère inconnu à découvrir: 1-117 (Le Rocher, Monaco).

Schevill, W. \& B. Lawrence, 1953. Auditory response of a Bottlenosed Porpoise (Tursiops truncatus) to frequencies above 100 kc. J. exp. Zool., 124: 147-164.

Sokolov, V.E., O.F. Chernova, Z.P. Zinkevich \& G.V. Khakhin, 1976. O khimicheskoKZi akkusticheskoKZkommunikatskii vykhukholi. Upravlenie povedeniem zhivotnykh: 369-370 (Moskva).

Sokolov, V.E., O.F. Chernova, Z.P. Zinkevich \& G.V. Khakhin, 1977. Spetsificheskaya podkhvostovaya zheleza vykhukholi (Specific subcaudal gland of Desmana moschata). Zool. Zh., 56(2): 250-256 (russisch, mit englischem Summary).

Stephan, H. \& R. Bauchot, 1968. Gehirn und Endocranialausguß von Desmana moschata. Morph. Jb., 112(2): 213225.

Stephan, H., G. Baron \& H.D. Frahm, 1991. Comparative brain research in mammals, 1. Insectivora: 1-573 (Springer Verlag, Berlin/Heidelberg/New York).

Weber, M., 1904. Die Säugetiere. Einführung in die Anatomie und Systematik der recenten und fossilen Mammalia: 363 (Gustav Fischer, Jena).

Eingegangen am 26. Juli 1995 